\title{
Myocardial architecture and patient variability in clinical patterns of atrial fibrillation
}

\author{
Kishan A. Manani, ${ }^{1,2,3}$ Kim Christensen, ${ }^{1,3}$ and Nicholas S. Peters ${ }^{2}$ \\ ${ }^{1}$ The Blackett Laboratory, Imperial College London, London SW7 2BW, United Kingdom \\ ${ }^{2}$ National Heart and Lung Institute, Imperial College London, London W12 ONN, United Kingdom \\ ${ }^{3}$ Centre for Complexity Science, Imperial College London, London SW7 2AZ, United Kingdom \\ (Received 27 January 2016; revised manuscript received 31 May 2016; published 4 October 2016)
}

\begin{abstract}
Atrial fibrillation (AF) increases the risk of stroke by a factor of 4-5 and is the most common abnormal heart rhythm. The progression of AF with age, from short self-terminating episodes to persistence, varies between individuals and is poorly understood. An inability to understand and predict variation in AF progression has resulted in less patient-specific therapy. Likewise, it has been a challenge to relate the microstructural features of heart muscle tissue (myocardial architecture) with the emergent temporal clinical patterns of AF. We use a simple model of activation wave-front propagation on an anisotropic structure, mimicking heart muscle tissue, to show how variation in AF behavior arises naturally from microstructural differences between individuals. We show that the stochastic nature of progressive transversal uncoupling of muscle strands (e.g., due to fibrosis or gap junctional remodeling), as occurs with age, results in variability in AF episode onset time, frequency, duration, burden, and progression between individuals. This is consistent with clinical observations. The uncoupling of muscle strands can cause critical architectural patterns in the myocardium. These critical patterns anchor microreentrant wave fronts and thereby trigger AF. It is the number of local critical patterns of uncoupling as opposed to global uncoupling that determines AF progression. This insight may eventually lead to patient-specific therapy when it becomes possible to observe the cellular structure of a patient's heart.
\end{abstract}

DOI: 10.1103/PhysRevE.94.042401

\section{INTRODUCTION}

A key challenge in the mathematical modeling of diseases is to link microscopic variation in individuals (e.g., genetic, metabolic, or tissue structure) to variation in disease outcomes [e.g., the occurrence, recurrence, or persistence of atrial fibrillation $(\mathrm{AF})]$. In this paper we show how variation in microstructure affects the behavior of AF, suggesting a single mechanism for the origin of clinically observed variability of AF behavior. Atrial fibrillation is characterized by the apparently random propagation of multiple activation wave fronts in atrial muscle (myocardium). This gives rise to $\mathrm{AF}$ episodes of variable duration. Typically, short self-terminating episodes become longer with time until they do not terminate spontaneously. Current clinical guidelines (American College of Cardiology, American Heart Association, and European Society of Cardiology) define AF by the episode duration as paroxysmal (less than seven days), persistent (more than seven days), long-standing persistent (more than one year), and permanent (clinical decision to not treat) [1]. However, AF episodes will in fact lie on a continuum of durations. The natural history of AF is usually discussed using this classification scheme, which by its technical definition allows for progression of paroxysmal to persistent but not the reverse. However, this classification scheme becomes problematic in cases where episodes lasting longer than seven days terminate and are followed by episodes shorter than seven days, which is observed to occur frequently in patients [2]. Indeed, Sugihara et al. [3] could not consistently apply these guidelines to

Published by the American Physical Society under the terms of the Creative Commons Attribution 3.0 License. Further distribution of this work must maintain attribution to the author(s) and the published article's title, journal citation, and DOI. their continuously monitored patients because AF episode durations were observed to remit from more than seven days to less than seven days. Hence, they defined a different classification scheme based on AF burden (fraction of time in $\mathrm{AF}$ ) to describe their observations rather an arbitrary seven-day cutoff to distinguish between patients. It has been suggested that AF induces atrial electrophysiological changes (e.g., action potential duration shortening) and microstructural changes (e.g., fibrosis or gap junctional uncoupling), which promote further AF. This self-perpetuation has been termed $\mathrm{AF}$ begets $\mathrm{AF}$ [4]. While fibrosis promotes $\mathrm{AF}$, the quantitative relationship and the mechanism by which fibrosis promotes $\mathrm{AF}$ are not fully understood [5].

Sugihara et al. [3] monitored AF patients continuously in a long-term study (1031 cumulative patient years, mean 3.2 years per patient) using dual chamber permanent pacemakers. It was observed that progression to persistent $\mathrm{AF}$ was not inevitable, that is, some patients remained paroxysmal for the duration of the long-term follow-up and some patients' AF burden (fraction of time in $\mathrm{AF}$ ) could remit from $100 \%$ to less than $100 \%$ and relapse to $100 \%$ again. Indeed it has been observed that some patients do not progress from paroxysmal to persistent $\mathrm{AF}$, using current clinical guidelines, after as many as 22 years [6]. Veasey et al. [2] also used continuous monitoring data to show that after a mean seven-year followup, 35\% of patients that were initially classified as persistent AF using the current clinical guidelines were reclassified as paroxysmal AF. Other research has shown that the time course of $\mathrm{AF}$ is seen to vary between patients with similar fibrosis burden: Some patients progress rapidly from paroxysmal AF to persistent AF (on the order of months), while other patients do not progress at all (measured over decades) [6]. Furthermore, patients with a high fibrosis burden can remain paroxysmal and those with low fibrosis burden can be in persistent $\mathrm{AF}$ [6-9]. 
Thus we have the following recent clinical observations: (1) AF burden does not inexorably increase and can even spontaneously decrease, therefore not all patients appear to progress to persistent AF; (2) persistent $\mathrm{AF}$ can remit to paroxysmal AF, and (3) the common conception that fibrosis correlates with AF progression needs to be reconciled with the observed variability in AF burden for patients with similar levels of fibrosis. Individually and collectively these studies challenge the contemporary view of how AF evolves. Although it has been suggested that different pathological processes (mitral valve disease, diabetes, etc.) occurring in different patients may contribute to variability in AF progression [10], it is fair to say that there is no understanding of what causes the clinical observations summarized above.

The clinical patterns of AF are studied in the domain of populations on long time scales (months to years), whereas the microstructure of myocardium is often studied in wet laboratories within the domain of cellular electrophysiology on short time scales (from seconds to hours). These two vastly different time scales cannot be related experimentally. Similarly, many models of AF are computationally intensive due to their complexity. As a result, only short time periods (seconds or minutes) have been investigated. Thus, these models cannot address questions pertaining to the long time scales of disease progression. Previous work by Chang et al. [11] has explored the two time scales by modeling AF as a simple binary process that flips between normal sinus rhythm (SR) and arrhythmia at patient-specific rates. However, this study does not address the question of the microscopic origin of variability in clinical observations.

We use a very simple computational model to link the two time domains. The model is specifically designed to address the hypothesis that the stochastic nature of transversal uncoupling is an important factor in the temporal patterns of AF. We model a patient by simulating patient-specific tissue using a simple stochastic process and then assess the resulting temporal AF patterns.

The incidence of AF increases with age and is strongly associated with the accumulation of fibrosis [5]. In this paper we propose that the clinically observed diversity in AF progression can be caused by a single process, the progressive stochastic accumulation of transversal cellular uncoupling. Using a simple computer model, we show that different time courses of AF can occur between patients despite a similar degree of transversal cellular uncoupling. Thus the model provides an explanation to the aforementioned clinical observations, namely, that (1) the time course of AF progression can vary significantly between patients, (2) persistent AF can remit to paroxysmal $\mathrm{AF}$, and (3) macrostructurally similar myocardium can show very different AF behavior as a result of these microstructural differences. In addition to this, the model identifies specific critical architectural patterns of uncoupling between myocytes as the primary cause of AF induction. When access to the microstructure becomes available in the future this insight has the potential to result in patient-specific therapy.

\section{MODEL}

We have previously developed a model in which the activation wave fronts propagate on an anisotropic structure mimicking the branching network of heart muscle cells [12] (see Appendix A for a complete description of the model).
The tissue is represented by an $L \times L$ square grid of discrete cells where each cell is always coupled to its longitudinal neighbors but with probability $v$ to its transversal neighbors. This generates a lattice with anisotropic coupling, mimicking the uncoupling of transversal cell-to-cell connectivity through the parameter $v$. We use the simplest model of cell kinetics to mimic the action potential so that a cell may be in one of three states: resting (repolarized), excited (depolarizing), or refractory. An excited cell causes neighboring coupled resting cells to become excited. Thus the wave front is a coherent propagation of this excitation through the simulated tissue.

For each "patient" the initial conditions are created by assigning the same number of vertical connections (identical initial $v$ ) but at different random positions. Next the accumulation of transversal cellular uncoupling is implemented by reducing $v$ (e.g., to mimic the progression of fibrosis or gap junctional uncoupling). To do this, we run simulations for a period of $T=4.3 \times 10^{7}$ time steps in the computer model and vertical connections are removed at a rate of one connection every 9000 time steps. We note that the actual rate at which transversal uncoupling accumulates in humans is unknown and may differ between patients. Hence, the rates used in the model are set to be identical between simulated patients with the aim of capturing the generic phenomenon of the accumulation of uncoupling over time thought to occur frequently in humans [6]. We observe the dynamics of activation wave fronts as the transversal uncoupling accumulates in the tissue. All other model parameters are set to physiological values as described in [12] (see Appendix A).

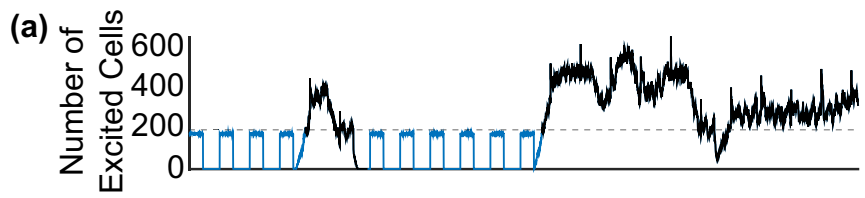

(b)

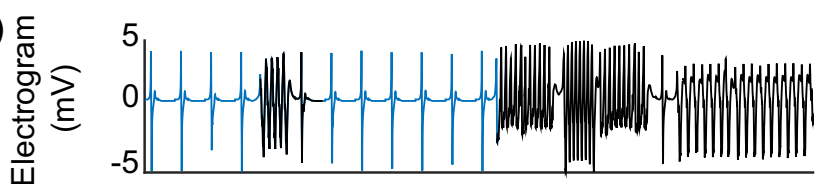

(c)

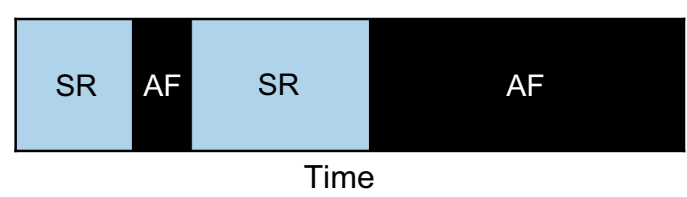

FIG. 1. Numerical simulation for a $200 \times 200$ system where pacemaker cells activate periodically. (a) During sinus rhythm (SR, blue curve) the number of excited cells varies with the same period as the pacemaker cells. When the number of excited cells exceeds a threshold (220, see the dashed gray line) it implies that the system is in fibrillation (AF, black curve). The system is defined to return to SR when the system is below threshold for more than one normal sinus rhythm beat. (b) A rectangular electrode of size $1 \mathrm{~mm}^{2}(10 \times 10$ cells $)$ placed at the center of the tissue is used to simulate the electrogram. During fibrillation (black curve) the rate of the electrogram increases by a factor of 2-5. (c) Associated binary signal of the time series in (a) into periods of SR (blue filled area) and AF (black filled area). Over long-time simulations, the AF burden can be computed from this as the fraction of time in $\mathrm{AF}$. 


\section{RESULTS}

We ran 32 lattice simulations, representing 32 patients, with the same initial fraction of vertical connections distributed randomly in each simulated tissue. We start at $v=0.25$ where all the simulated heart muscle tissue are in SR but when lowering $v$, fibrillation may emerge. The number of excited cells can be used to determine when the system is in fibrillation (see Fig. 1, for example, and the associated electrogram) and hence determine the associated AF burden, which we define as the amount of time in AF divided by the total observed time. To define paroxysmal and persistent AF in the model we use a scheme similar to that of Sugihara et al. [3] based on AF burden. That is, we call periods of AF burden being less than $100 \%$ paroxysmal AF and burden of $100 \%$ persistent AF. Permanent AF traditionally refers to the clinical decision not to treat and thus is not informative of the dynamics of AF.

Figure 2 shows the time course of four particular simulated patients. Patient A undergoes what would be considered the standard progression from paroxysmal AF, with low AF burden, to persistent AF, with maximal AF burden. Patient B, however, shows isolated short-lived episodes of paroxysmal AF with a sudden crossover to persistent AF. Hence, patient $\mathrm{B}$ lacks a gradual progression from paroxysmal AF to persistent AF. Patient $\mathrm{C}$ also had a few isolated episodes

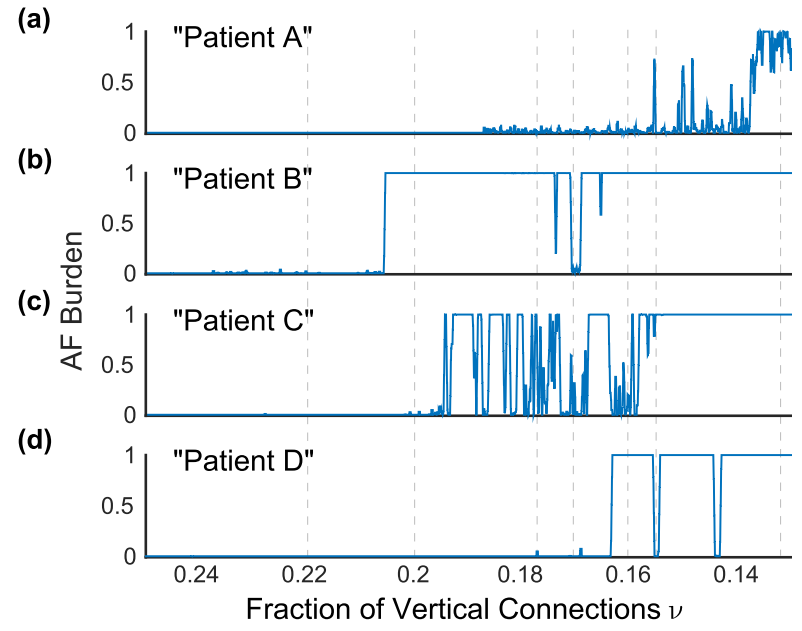

FIG. 2. The AF burden varies with time, calculated from the time in $\mathrm{AF}$ in a sliding window of $5 \times 10^{6}$ time steps for four different patients. Each simulation begins with an initial fraction of vertical connections of $v=0.25$ and is depleted to $v=0.13$, mimicking progression of stochastic uncoupling over a simulated time period of $4.3 \times 10^{7}$ time steps. (a) Patient A develops paroxysmal AF for $v \lesssim$ 0.188 , which eventually develops into persistent AF at $v \lesssim 0.138$. (b) Patient B develops short-lived episodes of AF for $0.206 \lesssim v \lesssim$ 0.238 and a sudden transition into persistent $\mathrm{AF} v \approx 0.206$. This relapses into paroxysmal $\mathrm{AF}$ three times before remitting back into persistent $\mathrm{AF}$ at $v \approx 0.166$. (c) Patient $\mathrm{C}$ shows a phase of relapsing-remitting paroxysmal to persistent $\mathrm{AF}$ for a considerable period of time until AF becomes persistent at $v \approx 0.159$. (d) Patient $\mathrm{D}$ shows isolated short-lived episodes of AF and a sudden transition into persistent $\mathrm{AF}$ at $v \approx 0.164$. Remission back into sinus rhythm occurs twice ( $v \approx 0.156$ and $v \approx 0.145$ ) before AF becomes persistent. The vertical dashed lines are values of fractions of vertical connections at which simulations are rerun without progressive uncoupling, that is, with a fixed fraction of vertical connections (see Fig. 3). of paroxysmal AF before entering a much more disordered relapsing-remitting phase between paroxysmal and persistent AF with different AF burdens compared to patient B. Patient D underwent a sudden transition from sinus rhythm to persistent $\mathrm{AF}$, but has phases of sinus rhythm interrupting persistent $\mathrm{AF}$ during the time course of the disease.

These four patients are archetypes of the time course of AF that we observe in our simulations. In addition to the variability in the progression to persistent $\mathrm{AF}$ we note that the onset of $\mathrm{AF}$ occurs at significantly different amounts of uncoupling, that is, fractions of vertical connections. These findings are consistent with the clinical observations that macroscopically similar myocardium can show large variability in AF burden $[3,6]$.

Furthermore, we observe that AF activity tends to change suddenly. That is, the frequency and duration of AF episodes change rapidly rather than gradually with progressive uncoupling (see Fig. 3). This is consistent with the clinical observation that macrostructurally similar myocardia (e.g., quantified by the global average fibrosis burden) show very different AF characteristics [6]. The frequency and duration of AF events are different in each simulated patient. However, in addition to clinical studies we can identify the microscopic origins of the observed behavior.

The differences in the behavior of these simulations are explained to a large degree by the number of localized critical regions with specific architectural patterns of coupling observed in the simulated tissues. In the model we detect these critical regions by detecting complete loops of wave-front activation, that is, microreentrant circuits. However, these critical patterns of uncoupling might also be determined structurally as these local patches of tissue have connections

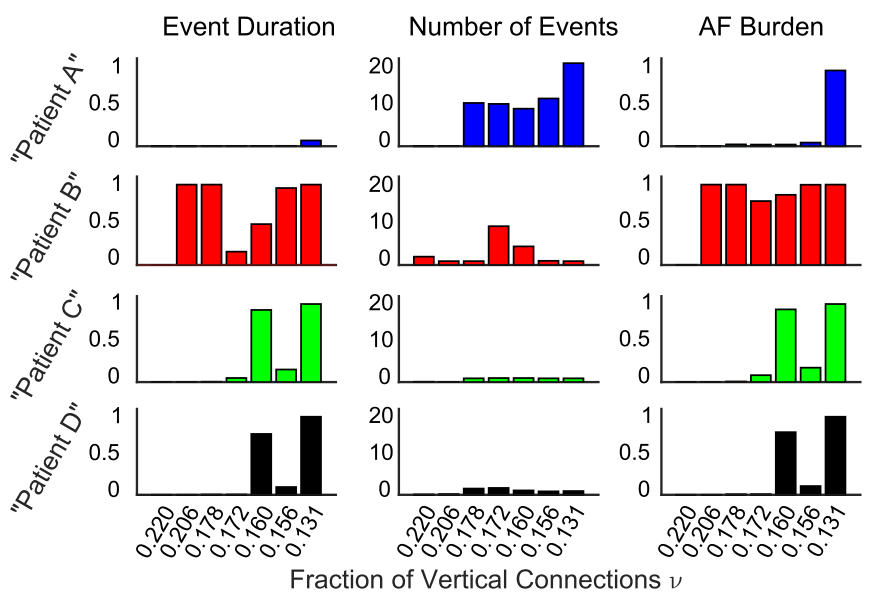

FIG. 3. Seven snapshots at $v=0.220,0.206,0.178,0.172,0.160$, 0.156 , and 0.131 of variability in event duration (expressed as a fraction of the simulation time $1.2 \times 10^{7}$ time steps), number of $\mathrm{AF}$ events, and AF burden for repeated simulations as a function of fraction of vertical connections. We rerun the simulations shown in Fig. 2 for $1.2 \times 10^{7}$ time steps starting from particular values of the fraction vertical connections (see the vertical dashed lines in Fig. 2) in which at least one of the four simulations displayed non-sinus-rhythm behavior. Note that we see variability both within a patient as the time course of AF progresses and between patients. These three observables of event duration, number of AF events, and AF burden are seen to vary significantly between real patients as well [3]. 
and dysfunctional cells arranged in a configuration that allows the formation of pinned microreentrant circuits [12]. These regions are characterized by large contiguous regions of uncoupled cell akin to the obstructive fibrosis found to promote AF in goats [13]. We observe that the first occurrence of AF coincide with the (chance) emergence of the first critical structure at $v=0.188,0.238,0.228$, and 0.177 for patients A, B, C, and $\mathrm{D}$, respectively (see Fig. 4). Furthermore, the number of these critical regions varies between patients despite having the same fraction of vertical connections (macroscopic measure). It is the variation in the number of these critical regions that causes the variability in the observed AF behavior shown in Figs. 2 and 3 [see Fig. 4(e)] (see also the full set of 32 patients in Appendix B). We note that at least one critical region is needed

(a)

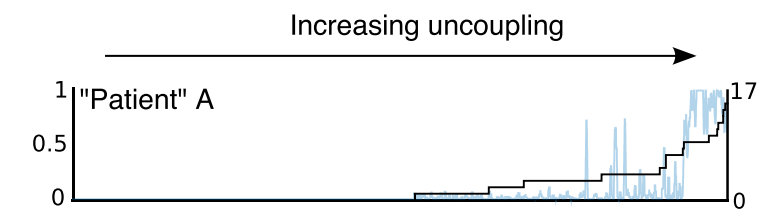

(b)

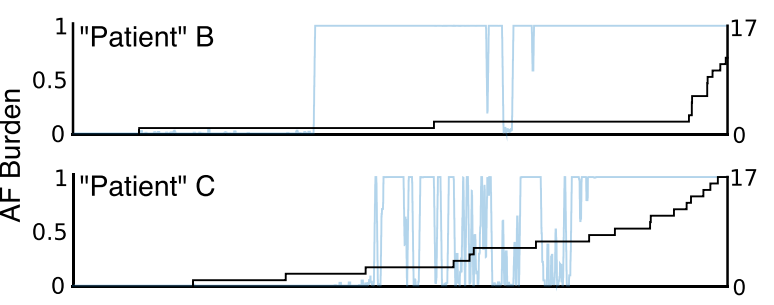

(d)

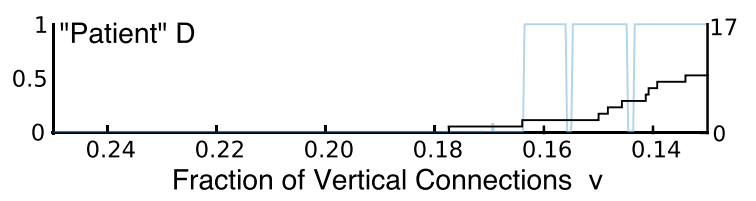

(e)

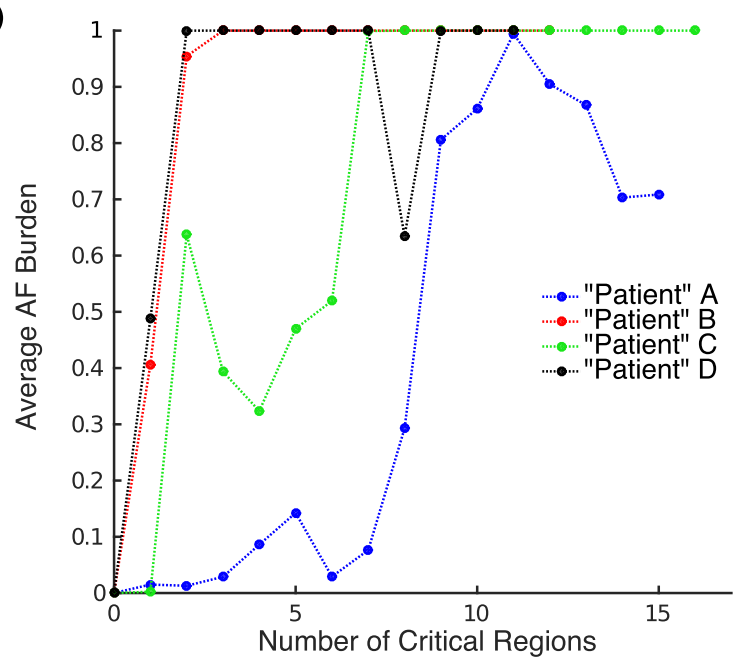

FIG. 4. (a)-(d) Accumulation of initiating critical regions (black curve) versus the fraction of vertical connections for patients A-D. The onset of AF in each patient coincide with the first appearance of a critical region. As uncoupling progresses (the fraction of vertical connections is reduced), the number of initiating critical regions increases. (e) Average AF burden for each patient as a function of the number of critical regions. The differences in the accumulation of critical regions between patients better predict the variability in the AF behavior observed. to initiate AF in the model, however, the average AF burden increases nonlinearly, reflecting an increase in episode duration, as the number of critical regions increases [see Fig. 4(e)].

\section{CONCLUSION}

The progression of AF and its variability is poorly understood. Fibrosis, among other factors, is known to be important. However, the reason why so much heterogeneity in AF behavior occurs in patients with similar fibrosis burden is not known. In addition to this, insights from studies in cardiac tissue slices (short time scales) have yet to be reconciled with the clinical patterns of AF development (long time scales). We bridge this gap using computational modeling and identify how structural characteristics of myocardium and uncoupling alone can give rise to patient variability. We note that the variability in the simulated patients is strictly due to specific architectural patterns of vertical uncoupling between cells. Fibrosis is one mechanism of cellular uncoupling. An additional mechanism of uncoupling is gap junctional remodeling, whereby the passive high resistance pathways between cells are redistributed to enhance anisotropic conduction and may result in the failure of action potential propagation [14-16]. Indeed, gap junctional remodeling is known to be arrhythmogenic.

We have shown that a simple model of heart muscle tissue can display the clinically observed variability in AF progression. This variability originates from the chance occurrence of critical regions characterized by poor vertical connectivity. The model reproduces clinical observations: variability in AF episode onset time, frequency, duration, and progression (Fig. 3) along with (1) significant variability in the time course of AF progression between patients, (2) persistent AF remitting to paroxysmal AF, and (3) macrostructurally similar myocardium that can show very different AF behavior (Figs. 2 and 4). Thus we show that a single pathological mechanism, namely, uncoupling, can result in patterns of AF observed clinically. Specific architectural patterns of uncoupling rather than the global uncoupling (e.g., total fibrosis burden) were observed to drive AF. Thus, our work suggests that the tissue microstructure is essential in determining the time course of $\mathrm{AF}$ in a given patient. This is a first step in relating structural features of myocardium, greatly studied in a basic science context, to patterns of AF in patients, studied in a clinical context. When experimental access to in vivo tissue microstructure becomes available in the future, insight from this work might potentially lead to patient-specific therapy.

\section{ACKNOWLEDGMENTS}

We are very grateful to Nick Linton for his pertinent comments regarding the manuscript. This work was supported by the British Heart Foundation (Grant No. RG/10/11/28457), the ElectroCardioMaths Programme of Imperial BHF Centre of Research Excellence, and the National Institute for Health Research Biomedical Research Centre. K.A.M. and K.C. conceived the experiment and its interpretation. K.A.M. performed the simulations and created the figures. N.S.P. provided essential comments in relating the model to a clinical context. K.C. and N.S.P. supervised the project. All authors discussed the results and commented on and contributed to the writing of the manuscript. 


\section{APPENDIX A: FULL DESCRIPTION OF THE MODEL}

We provide a brief summary of the model and parameters used. The tissue is represented by an $L \times L$ square grid of discrete cells. Heart muscle tissue forms a branching network of interacting cells where each cell is coupled to its neighbors longitudinally and to its neighbors transversally with probability $v$. The structure of the lattice can be considered to be a set of aligned longitudinal cables of single cell thickness that are coupled to neighboring cables with frequency $v$. The lateral uncoupling of cells is thus represented by reducing the frequency of vertical couplings through the parameter $v$. To represent the simplest features of an atrium we apply periodic boundary conditions vertically and open boundary conditions horizontally to the two-dimensional model, giving a cylindrical topology, which is a convenient substrate to study the evolution of wave fronts. Cells along the left boundary are pacemakers and self-excite at a fixed period $T^{\prime}=660 \mathrm{~ms}$ to mimic normal cardiac rhythm.

Simplifying the action potential, a cell may be in one of three states: resting (repolarized), excited (depolarizing), or refractory. An excited cell causes neighboring coupled resting cells to become excited. Thus the wave front is a coherent propagation of this excitation throughout the tissue. A healthy atrial area is $L^{\prime} \times L^{\prime} \approx 20 \mathrm{~cm}^{2}$. Atrial muscle tissue is made of nearly cylindrical myocytes of length $\Delta x^{\prime} \approx 100 \mu \mathrm{m}$ and diameter $\Delta y^{\prime} \approx 20 \mu \mathrm{m}$ packed in an irregular brick-wall-like pattern. The time taken for a cell to depolarize, $\Delta t^{\prime} \approx 0.6 \mathrm{~ms}$, is much shorter than the refractory period $\tau^{\prime} \approx 150 \mathrm{~ms}$.

Cellular electrical dysfunction of any cause can introduce a degree of noise into the excitation process. A fraction of randomly chosen cells in the lattice is chosen to be dysfunctional. Such cells have a finite probability of not exciting in response to an excited neighbor and hence can block the propagation of activation.

Translating real tissue values into the model gives $L=$ $L^{\prime} / \Delta x^{\prime}=1000$. We coarse grain the model by taking $\Delta x^{\prime} \rightarrow$ $b \Delta x^{\prime}$, where $b$ is the number of cells within a unit of space in the model. As the conduction velocity $\theta_{x}^{\prime}=\Delta x^{\prime} / \Delta t^{\prime} \approx$ $0.2 \mathrm{~ms}^{-1}$ is fixed, we also find $\Delta t^{\prime} \rightarrow b \Delta t^{\prime}$. We take $b=5$, implying that the parameters of the model are system size $L=200$, refractory period $\tau=\tau^{\prime} / b \Delta t^{\prime}=50$, the fraction of dysfunctional cells $\delta=0.05$, the probability of dysfunction $\epsilon=0.05$, and the period of pacing $T=T^{\prime} / b \Delta t^{\prime}=220 \mathrm{using}$ $T^{\prime}=660 \mathrm{~ms}$, leaving the fraction of transverse connections $v$ as the only control parameter. See our previous paper [12] for a detailed discussion on the model.

\section{APPENDIX B: 32 SIMULATED PATIENTS}

We present the results of all 32 simulated patients. We start at $v=0.25$, where all the simulated heart muscle tissue is in SR, but as we lower $v$, fibrillation emerges. The initial conditions for each patient are created by assigning the same number of vertical connections (identical initial $v$ ) but at different random positions. Next the accumulation of cellular uncoupling is implemented by reducing $v$ (e.g., to mimic the progression of fibrosis or gap junctional uncoupling). To do this, we run simulations for a period of $T=4.3 \times 10^{7}$ time steps in the computer model and vertical connections are removed at a rate of one connection every 9000 time steps.

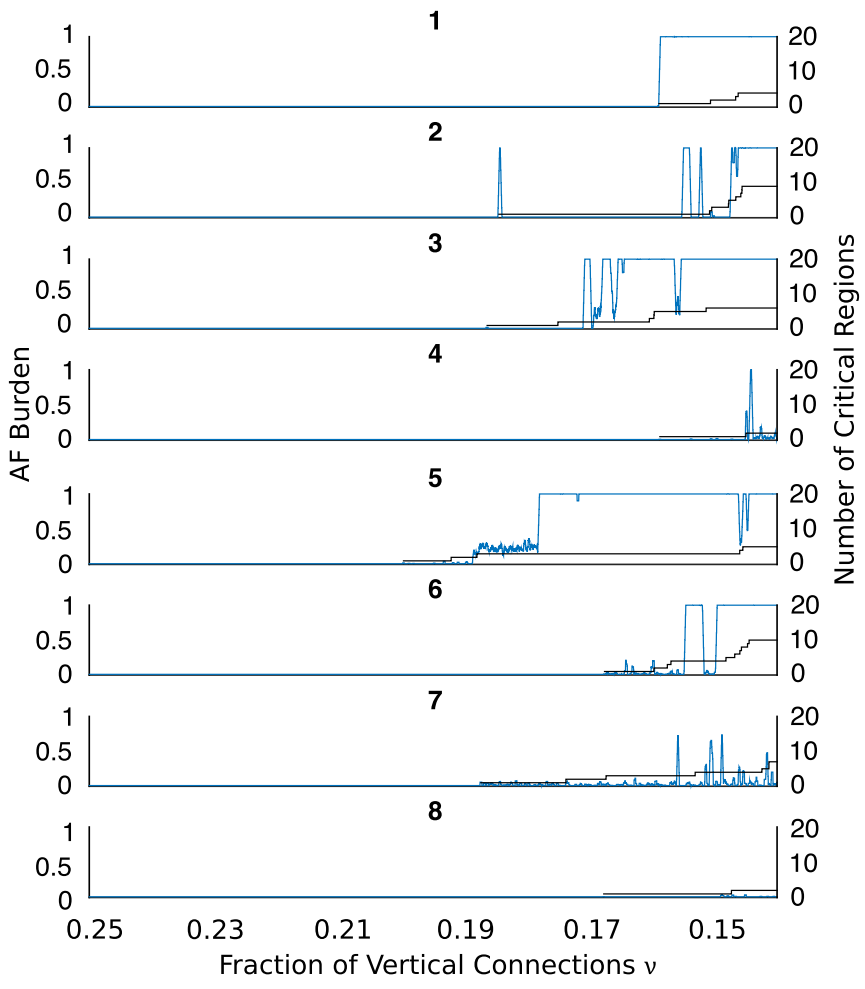

FIG. 5. The AF burden (blue) varies with time, calculated from the time in $\mathrm{AF}$ in a sliding window of $5 \times 10^{6}$ time steps for simulations 1-8. Also shown is the accumulation of initiating critical regions (black curve) versus the fraction of vertical connections.

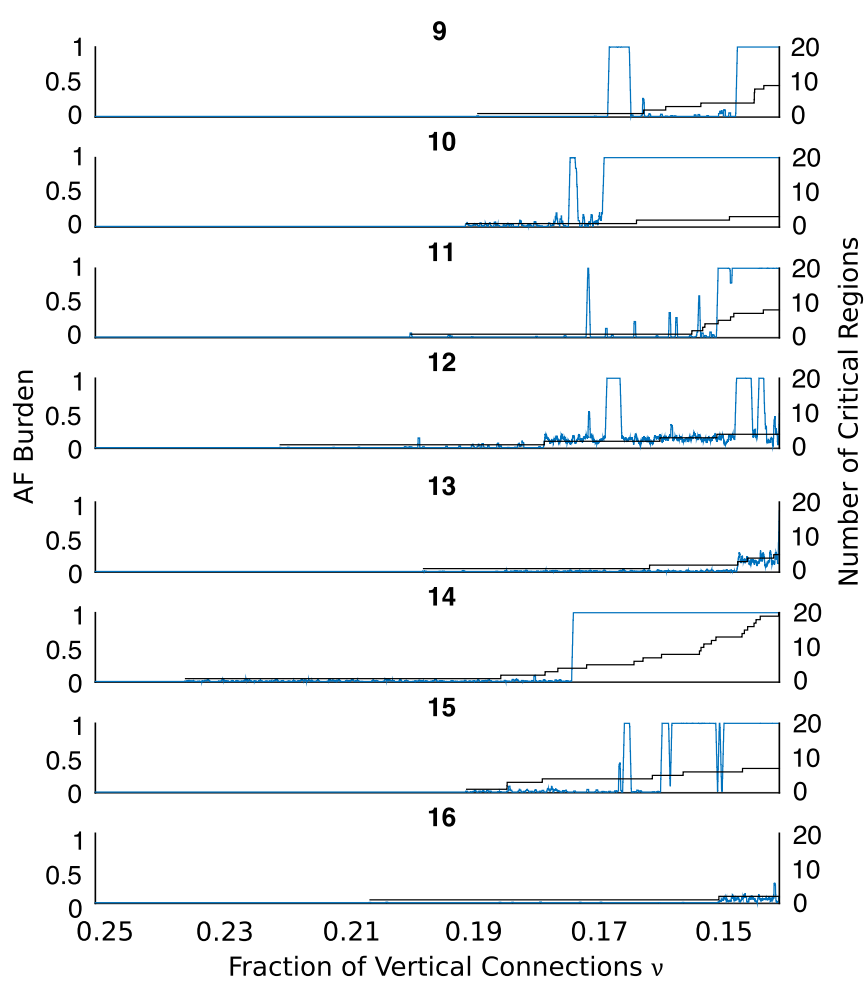

FIG. 6. The AF burden (blue) varies with time, calculated from the time in $\mathrm{AF}$ in a sliding window of $5 \times 10^{6}$ time steps for simulations 9-16. Also shown is the accumulation of initiating critical regions (black curve) versus the fraction of vertical connections. 


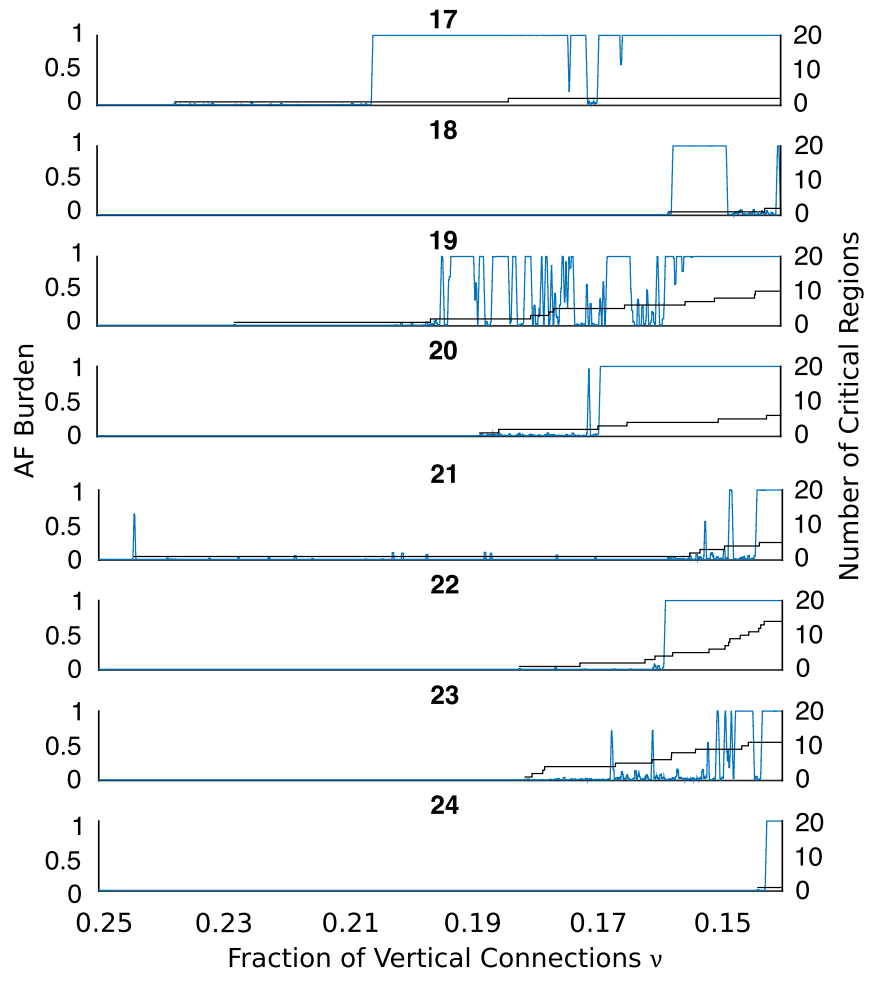

FIG. 7. The AF burden (blue) varies with time, calculated from the time in AF in a sliding window of $5 \times 10^{6}$ time steps for simulations 17-24. Also shown is the accumulation of initiating critical regions (black curve) versus the fraction of vertical connections.

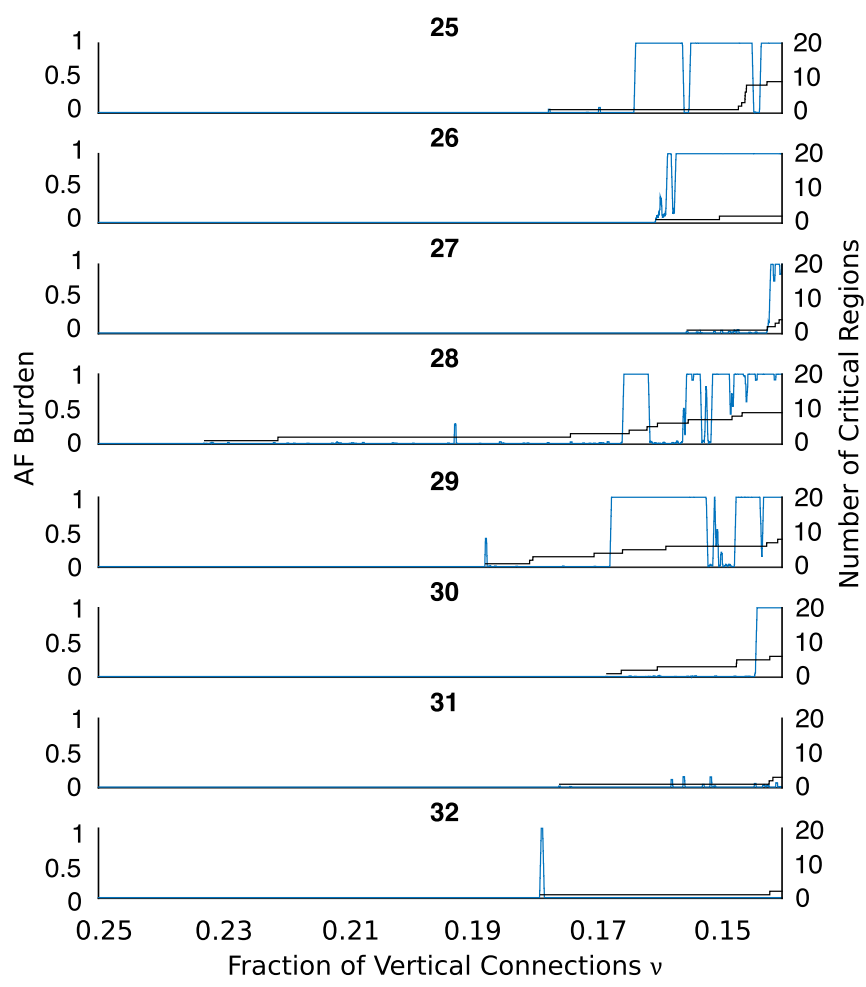

FIG. 8. The AF burden (blue) varies with time, calculated from the time in AF in a sliding window of $5 \times 10^{6}$ time steps for simulations 25-32. Also shown is the accumulation of initiating critical regions (black curve) versus the fraction of vertical connections.
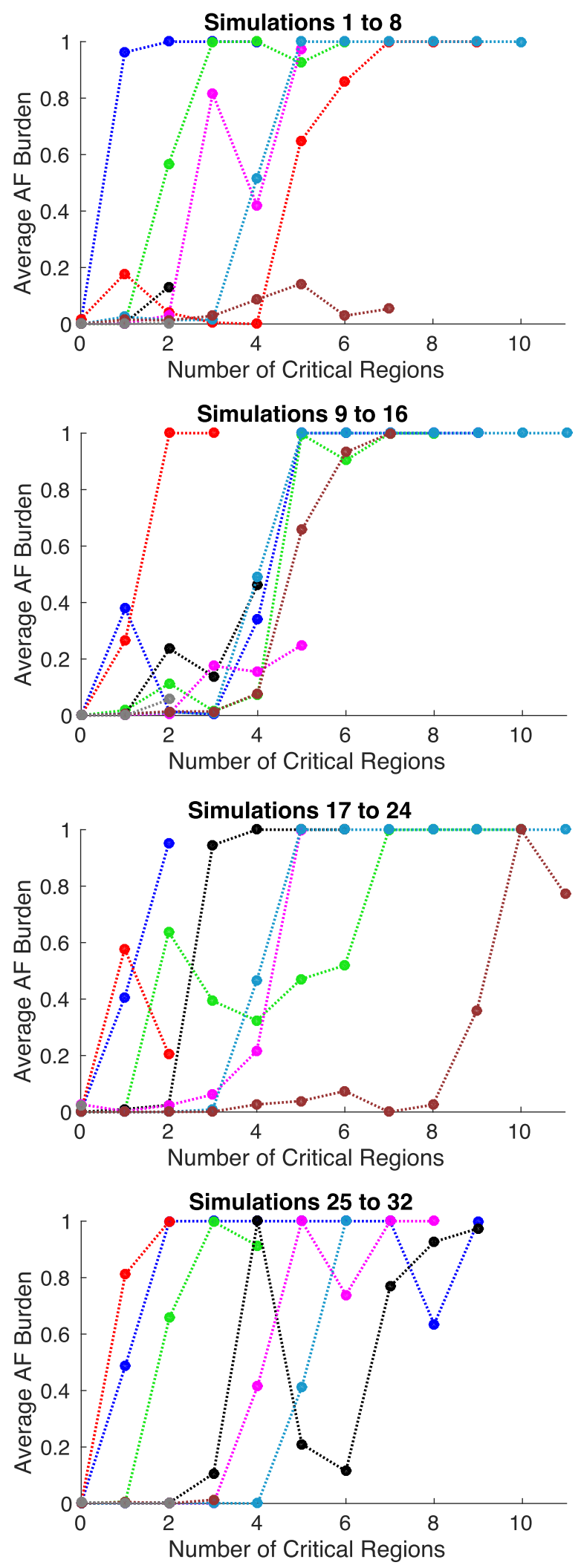

FIG. 9. Average AF burden for all 32 simulations as a function of the number of critical regions. The standard deviations and standard error are too small to be seen at this scale. The differences in the accumulation of critical regions between patients better predict the variability in the AF behavior observed. 
We observe the dynamics of excitation wave fronts as the transversal uncoupling accumulates in the tissue. All other model parameters are set to physiological values: $L=200$, $\tau=50, \epsilon=0.05$, and $\delta=0.05$. Pacemaker cells self-activate with a period of $T=220$.
The AF burden and number of critical regions versus fraction of vertical connections are shown in Figs. 1-4. We show the average AF burden as a function of the number of critical regions for each simulation in Figs. 5-9.
[1] A. J. Camm, P. Kirchhof, G. Y. Lip, U. Schotten, I. Savelieva, S. Ernst, I. C. Van Gelder, N. Al-Attar, G. Hindricks, B. Prendergast et al., Eur. Heart J. 31, 2369 (2010).

[2] R. A. Veasey, C. Sugihara, K. Sandhu, G. Dhillon, N. Freemantle, S. S. Furniss, and A. N. Sulke, J. Intervent. Cardiac Electrophysiol. 44, 23 (2015).

[3] C. Sugihara, R. Veasey, N. Freemantle, S. Podd, S. Furniss, and N. Sulke, Europace 17, 864 (2015).

[4] M. C. Wijffels, C. J. Kirchhof, R. Dorland, and M. A. Allessie, Circulation 92, 1954 (1995).

[5] S. De Jong, T. A. van Veen, H. V. van Rijen, and J. M. de Bakker, J. Cardiovasc. Pharm. 57, 630 (2011).

[6] H. Kottkamp, Eur. Heart J. 34, 2731 (2013).

[7] R. S. Oakes, T. J. Badger, E. G. Kholmovski, N. Akoum, N. S. Burgon, E. N. Fish, J. J. E. Blauer, S. N. Rao, E. V. R. Dibella, N. M. Segerson et al., Circulation 119, 1758 (2009).

[8] A. W. Teh, P. M. Kistler, G. Lee, C. Medi, P. M. Heck, S. J. Spence, P. B. Sparks, J. B. Morton, and J. M. Kalman, J. Cardiovasc. Electrophysiol. 23, 232 (2012).
[9] A. Boldt, U. Wetzel, J. Lauschke, J. Weigl, J. Gummert, G. Hindricks, H. Kottkamp, and S. Dhein, Heart 90, 400 (2004).

[10] U. Schotten, S. Verheule, P. Kirchhof, and A. Goette, Physiol. Rev. 91, 265 (2011).

[11] E. T. Chang, Y. T. Lin, T. Galla, R. H. Clayton, and J. Eatock, PLoS ONE 11, e0152349 (2016).

[12] K. Christensen, K. A. Manani, and N. S. Peters, Phys. Rev. Lett. 114, 028104 (2015).

[13] N. Angel, L. Li, R. S. MacLeod, N. Marrouche, R. Ranjan, and D. J. Dosdall, J. Cardiovasc. Electrophysiol. 26, 1352 (2015).

[14] M. S. Spach, J. F. Heidlage, P. C. Dolber, and R. C. Barr, J. Electrocardiol. 34, 69 (2001).

[15] M. S. Spach, J. F. Heidlage, P. C. Dolber, and R. C. Barr, Circ. Res. 86, 302 (2000).

[16] M. L. Hubbard, W. Ying, and C. S. Henriquez, Europace 9, vi20 (2007). 